\title{
Orientaciones para desarrollar programas de tabaquismo
}

\author{
LEONARDO VÉJAR M.*
}

\section{Guidelines for developing smoking cessation programs}

This paper presents useful ideas for developing a smoking cessation program, using the experience of the South East Metropolitan Health Service (SSMSO) Santiago, Chile, since 2007. The objective of this program is contribute to decrease the smoking rate, help people to quit smoking and improving the prognosis of tobacco-related conditions. The strategies used are: 1. Enforce the Tobacco Law in health centers; 2. Promote the application of ABC council in all health centers; 3. Develop a Hospital Tobacco Addiction Treatment Unit; 4. Develop Tobacco Treatment Units in Ambulatory Care Centers; 5. Prevent the relapse of the hospitalized patient who has had a heart attack; 6. Create appropriate training instances.

Key words: Smoking cessation; tobacco use disorders; behavior addictive; recurrence; ambulatory care.

\section{Resumen}

Este documento presenta ideas útiles para desarrollar un programa de tabaquismo, usando la experiencia del Servicio de Salud Metropolitano Sur Oriente (SSMSO), desde 2007. El objetivo de este programa es contribuir a bajar la tasa de tabaquismo, ayudar a las personas a dejar de fumar y mejorar el pronóstico de las afecciones derivadas del consumo del tabaco. Las estrategias utilizadas son: 1. Hacer cumplir la Ley de tabaco en los centros de salud; 2. Promover la aplicación de consejería breve de cesación del tabaco $(A B C)$ en todos los centros de salud; 3. Desarrollar una Unidad de Tratamiento de Adicción al Tabaco; 4. Desarrollar Unidades de Tratamiento del Tabaco en APS. 5. Prevenir la recaida del paciente hospitalizado que ha tenido un infarto; 6. Crear instancias de capacitación adecuada.

Palabras clave: Dejar de fumar; tabaquismo; comportamiento adictivo; atención ambulatoria, recurrencia.

\section{Introducción}

Se presentan las ideas que se consideraron útiles para desarrollar un programa de tabaquismo, utilizando la experiencia del SSMSO Región Metropolitana, desde los años 2007 a la fecha.

\section{Objetivos}

Contribuir a bajar la tasa de tabaquismo, ayudar a las personas a dejar de fumar y mejorar el pro- nóstico de las afecciones derivadas del consumo.

Estrategia 1. Hacer cumplir los artículos de la Ley del tabaco 20.660 atingentes a la prohibición de fumar en hospitales y centros de salud. El director del establecimiento de salud está facultado para prohibir fumar dentro del recinto hospitalario o para permitir fumar a los funcionarios y otras personas en áreas abiertas dentro del recinto del hospital (Ley 20.660 art 11 letra h). El objetivo de establecer esta restricción es que los funcionarios de la salud sean estimulados a dejar de fumar. En segundo lugar, se debe hacer extensiva

* Pediatra Especialista Broncopulmonar, Experto en Tabaquismo SEPAR.

Programa Tabaquismo Servicio de Salud Metropolitano Sur Oriente, Chile. 
esta indicación a los hogares de los pacientes.

Estrategia 2. Promover la aplicación de Consejería Breve (ABC-D). Esta actividad que puede contribuir a bajar la tasa de consumo en consultantes de los servicios de salud, para producir efectos debe ser aplicada en forma muy amplia y para ello se debe capacitar a todos los profesionales que tienen contacto con pacientes.

Estrategia 3. Formar una unidad de tratamiento de adicción al tabaco (UTAD). Una UTAD se ubica en el hospital base, y es un lugar donde se puede dar tratamiento intensivo de tabaquismo que comprende sesiones extensas de terapia con fármacos, con el objetivo de:

3.1 Detener el consumo en personas que presentan enfermedades asociadas a tabaquismo, cuya evolución y pronóstico se ve seriamente deteriorada por seguir fumando, estos pacientes son altamente dependientes de nicotina y generalmente requieren fármacos.

3.2 Ayudar a personas que solicitan ayuda para dejar de fumar, entre ellas a funcionarios de la salud, quienes deben ser los primeros en dejar de fumar por razones tales como: imagen, tiempo perdido en fumar, licencias y necesidad de prolongar su vida útil como funcionarios.

3.3 Docencia e investigación. Es un lugar adecuado para entrenar en terapia de cesación del tabaquismo y evaluar resultados.

\section{Pasos para el desarrollo de una UTAD}

Paso 1. Conocer información básica del establecimiento en que se instala el programa: población asignada, número de fallecimientos atribuibles a tabaco y cálculo del costo del tabaquismo.

Paso 2. Realizar una encuesta en el hospital para conocer el número de fumadores, características de la adicción, interés por dejar de fumar. Esta encuesta permite a la vez inscribir en el programa de tabaquismo a interesados en dejar de fumar.

Paso 3. Realizar reunión con los directores del servicio de salud, médicos y personal de salud para presentar la información demográfica y la obtenida en encuesta a los funcionarios. Además, señalar que el programa es costo-efectivo y que dejar de fumar es la actividad preventiva más potente de la salud. Finalmente, se solicitará apoyo para la formación de la unidad de tratamiento.

Paso 4. Formación de un equipo profesional con personas que deben tener capacitación avanzada en cesación del tabaquismo y vocación, lo que es independiente de la profesión.
Paso 5. Obtener recursos necesarios para la Unidad de Tratamiento.

Recurso humano: se requiere al menos 2 profesionales capacitados a los que se pueda asignar al menos de $6 \mathrm{~h}$ semanales a cada uno.

Recurso físico: una consulta para ingresar pacientes a tratamiento, una sala amplia para terapia grupal para al menos 15 personas. Un cooxímetro $\mathrm{y}$ un computador con acceso a internet.

Recursos financieros: costear un fármaco básico y uno alternativo. Los fármacos disponibles en Chile son bupropión, vareniclina y terapia de reemplazo de nicotina (TRN). El costo de bupropión, por tableta es aproximadamente $\$ 110$ y de vareniclina es de $\$ 580$. Se calculan 3 meses de tratamiento calculando una deserción de $50 \%$ de los pacientes.

Paso 6. Ingresar y monitorear información de pacientes: SSMSO tiene un sistema informático online (SISTAB). Que permite ingreso de datos de los pacientes a una ficha electrónica con seguimiento y cierre de casos Se puede ingresar datos desde todos los centros que participan en el programa, los que pueden ser monitoreados en eficiencia y rendimiento.

Estrategia 4. Crear Unidades de Tratamiento en atención primaria en salud (APS) hace necesario, cuando la población asignada es igual o mayor de 100.000 personas. Para desarrollar estas unidades se requiere seguir los mismos pasos antes señalados para una UTAD de hospital base, con la limitante que los centros de salud son dependientes de autoridades de salud comunales. Es recomendable asociar este desarrollo a programas bien estructurados, como respiratorio, cardiovascular o adicciones. La incorporación de tratamiento de tabaquismo a programas de Salud Mental y Adicciones ofrece la instancia de desarrollo más factible.

Estrategia 5: Evitar recaída del paciente hospitalizado que ha tenido un infarto. Muchos fumadores, que ingresan al hospital por infarto, salen de alta sin fumar y al poco tiempo recaen y reproducen sus riesgos originales. Este programa se inicia al detectar al paciente fumador en la Unidad Coronaria, aconsejarle el cese mediante $\mathrm{ABC}$, iniciar fármacos (vareniclina, TRN), ingresarlo al programa, continuar tratamiento después del alta UTAD, con seguimiento por 6 meses. Para desarrollar esta actividad, se requiere compartir los objetivos con los miembros del equipo de la Unidad Coronaria, obtener horas médicas, financiamiento de los fármacos y disponer la continuidad de la atención con acceso prioritario a la UTAD o centro APS.

Estrategia 6. Crear u ofrecer instancias de 
capacitación. Para las personas responsables de un programa de tabaquismo, se recomienda tomar cursos no inferiores a $140 \mathrm{~h}$, presenciales u onli$n e$, sea en el país (INTA, Universidad de Chile, Diplomado Finis Terrae) o en el extranjero (Curso Separ o Attud). Se recomienda ofrecer, cursos básicos (ABC-D y 4As) para profesionales APS y de Atención Secundaria, y cursos avanzados para los profesionales que deban realizar tratamiento intensivo y utilizar SISTAB.

\section{Bibliografía}

1.- VÉJAR L, MEDINA G, PONCE R, MORALES M, CASTILLO, POZO MJ, et al. Tratamiento de tabaquismo en funcionarios de la salud en 11 centros de Atención Primaria. Rev Prev Tab 2013; 15: 21-5.

2.- VÉJAR L, MEDINA G, MORALES M, et al. Intervención para cesación tabáquica en pacientes con EPOC Santiago de Chile. Rev Prev Tab 2013; 5: 1015. 